\title{
Potential Performance of SiC and GaN Based Metal Semiconductor Field Effect Transistors
}

\author{
H. Arabshahi* \\ Department of Physics, Ferdowsi University of Mashhad, \\ P.O. Box 91775-1436, Mashhad, Iran \\ (Received on 23 July, 2008)
}

\begin{abstract}
A Monte Carlo simulation has been used to model steady-state electron transport in $\mathrm{SiC}$ and $\mathrm{GaN}$ field effect transistor. The simulated device geometries and doping are matched to the nominal parameters described for the experimental structures as closely as possible. Simulations of SiC MESFETs of lengths 2, 2.6 and $3.2 \mu \mathrm{m}$ have been carried out and compared these results with those on GaN MESFETs of the same dimensions. The direct current $I V$ characteristics of the two materials were found to be similar, though the GaN characteristics were on the whole superior, reaching their operating point at higher drain voltages and possessing higher gains. However, oscillations in the drain current caused by changes in drain voltage in the GaN devices were not present to the same degree in the SiC devices. This difference is caused partially by the onset of the negative differential regime in $\mathrm{SiC}$ at a higher electric field than in $\mathrm{GaN}$ but the primary cause is the longer ballistic transport times in SiC. This suggests that GaN MESFETs may prove to have superior frequency response characteristics than SiC MESFETs.
\end{abstract}

Keywords: Ballistic transport; frequency response; Steady-state; Drain current

\section{INTRODUCTION}

$\mathrm{SiC}$ and $\mathrm{GaN}$ are wide bandgap semiconductors, and therefore have a high breakdown field and low thermal generation rate. These properties combined with good thermal conductivity and stability make them as an attractive material for high power, high temperature and radiation harsh environment electronic devices. Monte Carlo simulations pridict a peak electron velocity of $3 \times 10^{5} \mathrm{~ms}^{-1}$ and $2.3 \times 10^{5} \mathrm{~ms}^{-1}$ for $\mathrm{SiC}$ and $\mathrm{GaN}$ materials, respectively [1-2]. This makes possible high frequency operation devices.

The metal semiconductor field effect transistor (MESFET) is one of the most favoured devices in the construction of large scale integrated circuits because of its simplicity of construction, the comparative lack of dopant diffusion problems and the resultant high packing densities possible. Whilst the preferred semiconductor is still silicon, industry is now tooling up for wide band gap semiconductors like $\mathrm{GaN}$ or $\mathrm{SiC}$ production, which offers high electron mobility and hence the prospect of greater frequency operating rates. Their direct bandgap furthermore allows easier integration with optical devices. For this reason GaN MESFETs have received much attention in the literature, particularly with respect to their simulation [3-4] in an attempt to understand the basic principles of their operation. Monte Carlo methods have been used to a great extent in this effort because they allow an essentially exact solution of the Boltzmann transport equation and are subject only to statistical errors, unlike drift diffusion models which cannot accurately treat the hot-electron effects that are present to a high degree in $\mathrm{GaN}$ devices [5]. SiC offers the prospect of mobilities comparable to $\mathrm{GaN}$ and is increasingly being developed for the construction of optical switches. Other authors have also pointed out the potential importance of $\mathrm{SiC}$ and a few simple devices have been simulated [6]. This paper presents the results of a Monte Carlo simulation which is used to model electron transport in wurtzite GaN and SiC MESFETs including different tansistor lengths at room temperature. The simu-

*Electronic address: arabshahi@um.ac.ir lations have been carried out using a non-parabolic ellipsoidal valley model to describe transport in the conduction band.

This article is organised as follows. Details of the device fabrication model which is used in the simulated device are presented in section 2, and results for simulation carried out on $\mathrm{GaN}$ and SiC MESFETs are interpreted in section 3.

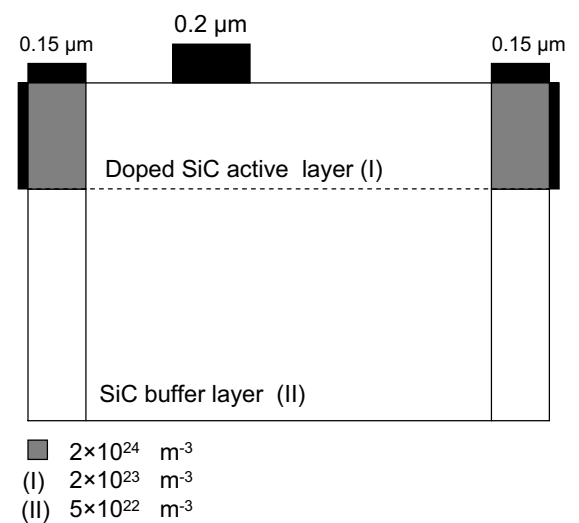

FIG. 1: The two-dimensional model of SiC/GaN MESFET. The modelled structure is divided into three regions, as indicated. Electron particles are initially distributed keeping all regions charge neutral. The location of the source and the drain implants and the top and back buffer layer are marked.

\section{MODEL DETAILS}

The Monte Carlo method may be used to simulate the motion of charge carriers through a semiconductor device by following the progress of several tens of thousands of test or representative superparticles. These particles are propagated classically between collisions according to their velocity, effective mass and the prevailing field. The selection of quantities such as the propagation time of a particle between scattering events or the mechanism by which the particle is to be scattered is done by generating a random number and using the number to choose the required quantity from predetermined tables of 


\begin{tabular}{|c|c|c|c|c|c|}
\hline Bulk material parameters & $\mathrm{SiC}$ & $\mathrm{GaN}$ & & & \\
\hline $\begin{array}{l}\text { Density } \rho\left(\mathrm{kgm}^{-3}\right) \\
\text { Longitudinal sound velocity } v_{s}\left(\mathrm{~ms}^{-1}\right) \\
\text { Low-frequency dielectric constant } \varepsilon_{s} \\
\text { High-frequency dielectric constant } \varepsilon_{\infty} \\
\text { Acoustic deformation potential } D(\mathrm{eV}) \\
\text { Polar optical phonon energy } \hbar \omega_{o p}(\mathrm{meV}) \\
\text { Bandgap } E_{g}(\mathrm{meV})\end{array}$ & $\begin{array}{c}3200 \\
1373 \\
9.7 \\
6.5 \\
15 \\
120 \\
3.2\end{array}$ & $\begin{array}{c}6150 \\
4330 \\
9.5 \\
5.35 \\
8.3 \\
99 \\
3.5\end{array}$ & & & \\
\hline Valley dependent parameters & $\Gamma_{1}$ & $U$ & $\Gamma_{3}$ & $M$ & $K$ \\
\hline $\begin{array}{l}\text { Effective mass }\left(\mathrm{m}^{*} / \mathrm{m}_{0}\right) \text { : } \\
\quad \mathrm{SiC} \\
\mathrm{GaN}\end{array}$ & $\begin{array}{c}0.28 \\
0.2\end{array}$ & $\begin{array}{c}0.39 \\
0.4\end{array}$ & $\begin{array}{l}0.6 \\
0.6\end{array}$ & $\begin{array}{l}0.57 \\
0.57\end{array}$ & $\begin{array}{c}0.54 \\
0.3\end{array}$ \\
\hline $\begin{array}{l}\text { Nonparabolicity }\left(\mathrm{eV}^{-1}\right) \text { : } \\
\text { SiC } \\
\text { GaN }\end{array}$ & $\begin{array}{c}0.32 \\
0.189\end{array}$ & $\begin{array}{c}0.5 \\
0.065\end{array}$ & $\begin{array}{l}0.029 \\
0.029\end{array}$ & $\begin{array}{l}0.0 \\
0.0\end{array}$ & $\begin{array}{c}0.03 \\
0.7\end{array}$ \\
\hline $\begin{array}{l}\text { Valley separation from } \Gamma_{1} \text {-valley }(\mathrm{eV}) \text { : } \\
\quad \mathrm{SiC} \\
\mathrm{GaN} \\
\text { Number of equvalet valley }\end{array}$ & $\begin{array}{c}0.0 \\
0.0 \\
1\end{array}$ & $\begin{array}{c}0.61 \\
2 \\
6\end{array}$ & $\begin{array}{c}1.3 \\
1.9 \\
1\end{array}$ & $\begin{array}{c}0.9 \\
3 \\
3\end{array}$ & $\begin{array}{c}0.67 \\
3.1 \\
2\end{array}$ \\
\hline
\end{tabular}

Table 1. Important parameters used in our simulations for wurtzite phase $\mathrm{SiC}$ and $\mathrm{GaN}$ materials.

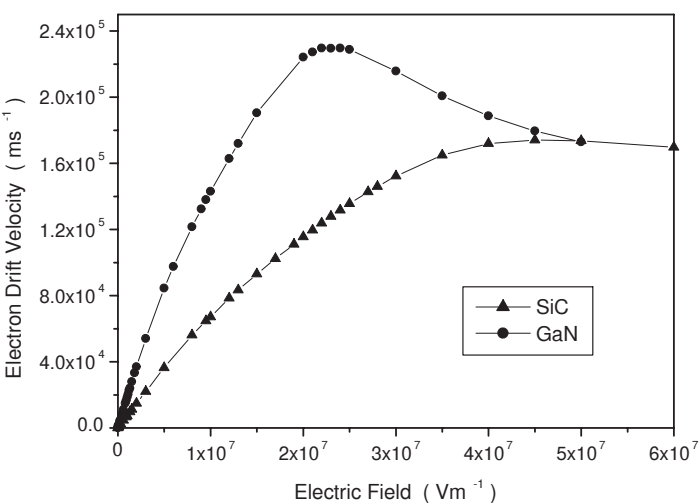

FIG. 2: Relationships between electron drift velocity and electric field for $\mathrm{GaN}$ (circles) and $\mathrm{SiC}$ (triangles) found using the parameters of table 1 .

probabilities for each process. Thorough discussions of the Monte Carlo method are given by Fawcett et. al [7] and Jacoboni et. al [8], and for a review see Moglestue et. al [9]. In this work, the two lowest energy conduction bands of the empirical pseudopotential band structure for wurtzite phase of $\mathrm{SiC}$ and $\mathrm{GaN}$ are chosen as a basis of an analytical multivalley conduction band model [10-13]. The pseudopotential band structure shows the conduction band minimum to be located at the $\Gamma$ point $\left(\Gamma_{1}\right)$, and lowest energy conduction band satellite valleys to occur at the $U$ point (located about two thirds of the way between the $L$ and $M$ symmetry points). Higher conduction band valleys are located at the $\Gamma$ point $\left(\Gamma_{3}\right)$, at the $M$ point, and at the $K$ point. In our Monte Carlo simulation, the two different $\Gamma$ valleys, the six equivalent $U$ valleys, the three equivalent $M$ valleys, and the two equivalent $K$ valleys, are rep- resented by ellipsoidal, nonparabolic dispersion relationships of the following form [8-9]

$$
E(\mathbf{k})\left[1+\alpha_{i} E(\mathbf{k})\right]=\frac{\hbar^{2}}{2}\left[\frac{k_{x}^{2}+k_{y}^{2}}{m_{\perp}^{*}}+\frac{k_{z}^{2}}{m_{\|}^{*}}\right]
$$

where $m_{\perp}^{*}$ and $m_{\|}^{*}$ are the transverse and longitudinal effective masses at the band edge and $\alpha_{i}$ is the non-parabolicity coefficient of the $i$-th valley. The material and valley parameters necessary for calculating the scattering probabilities used in the present Monte Carlo simulation are listed in table 1.

Electron particles in the employed ensemble Monte Carlo simulation occupy non-parabolic ellipsoidal valleys in reciprocal space, and obey Boltzmann statistics. Herring-vogt transformations are used to map carrier momenta into spherical valleys when particles are drifted, scattered or cross heterojunctions (where care has been taken to ensure that the crystal momentum in the plane of the junction is conserved across the interface). The electric field equations are solved self-consistently with the electron transport using a finite difference method [14], and the device grid potentials are updated at each ensemble drift timestep ( 1 femtosecond). Electrons in the bulk are scattered by ionised impurities and by bulk acoustic and non-polar optical phonon modes. Intervalley scattering by the absorption and emission of long wavelength acoustic and optic phonons have also been considered in the model. As described in detail [8-9], model devices are built up as a series of joined rectangular regions, with the electric field cell sizes matched along the join between each region. Each region can consist of multiple layers of different alloy composition and doping/compensation density.

The SiC/GaN MESFET can be described simply by three regions (figure 1), representing source and drain doping implants and a central region containing the supply layers. The field cell 

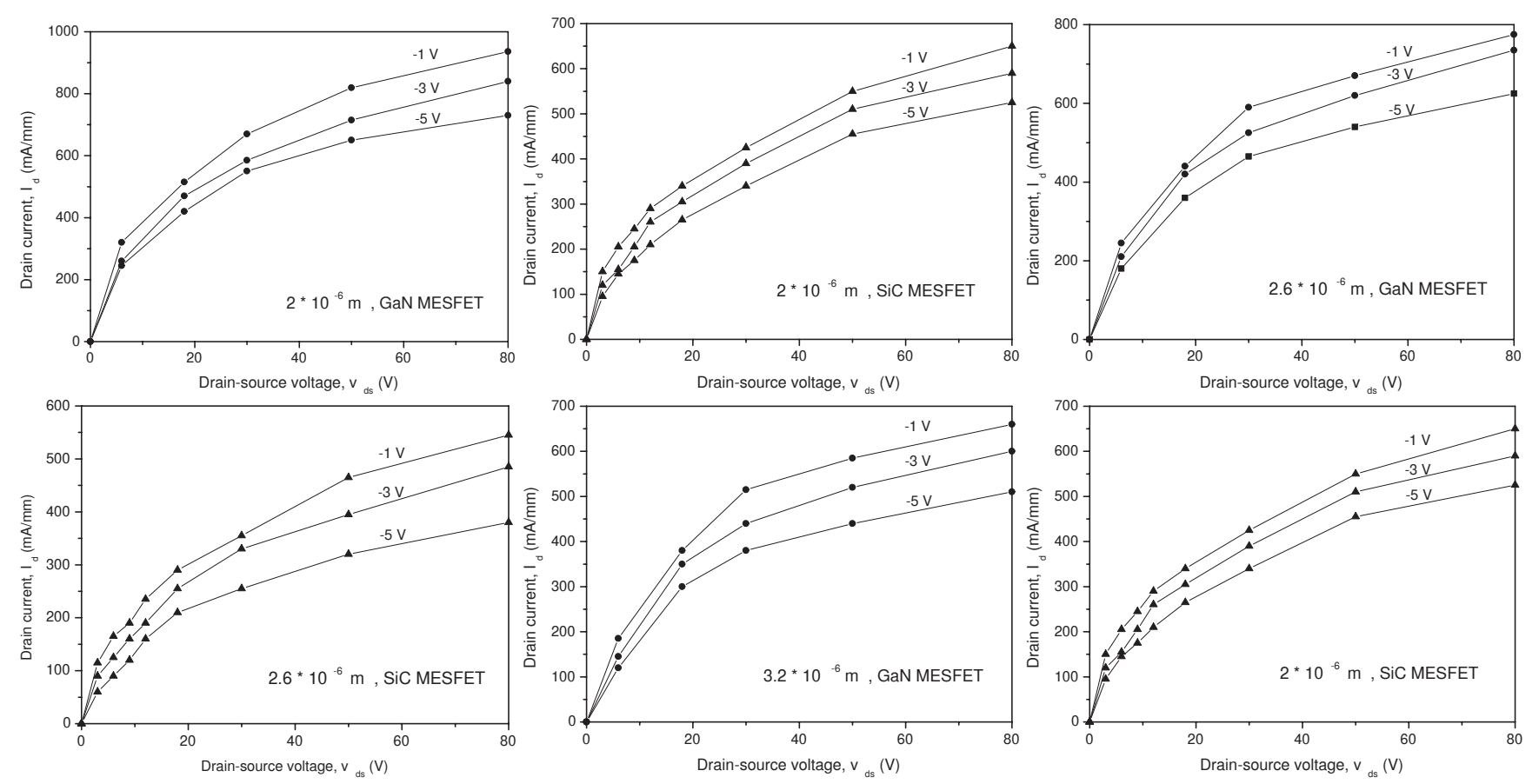

FIG. 3: Static $I V$ characteristics for GaN and SiC MESFETs for effective gate voltages of $-1 \mathrm{~V}$ to $-5 \mathrm{~V}$ and for different device length.

size used for the central region is $30 \mathrm{~nm}^{2}$ (horizontal $\times$ vertical), but that in the high doped source and drain implants is finer (10 $\mathrm{nm}^{2}$ ). Simulations of steady current characteristics have been carried out using 20000 electron paticles.

Figure 1 shows a schematic of the modelled SiC/GaN MESFET. The overall device length is $2 \mu \mathrm{m}$ in the $x$-direction and the device has a $0.2 \mu \mathrm{m}$ gate length and $0.15 \mu \mathrm{m}$ source and drain length. The source and drain have ohmic contacts and gate is in Schottky contact in $1 \mathrm{eV}$ to represent the contact potential at $\mathrm{Au} / \mathrm{Pt}$. The source and drain regions are doped to $2 \times 10^{24} \mathrm{~m}^{-3}$ electron concentration and the top and down buffer layers are doped to $2 \times 10^{23} \mathrm{~m}^{-3}$ and $5 \times 10^{22} \mathrm{~m}^{-3}$ electron concentration, respectively.

The drain current is obtained by counting the net charge flow through the drain contact. For the static $I V$ characteristics, the simulation was continued until the current converged to a value with an error of less than $1 \mathrm{~A}$ in two successive 10 ps intervals. The relationships between field and drift velocity obtained for each material are shown in figure 2 , where they are seen to compare well with [15-16].

\section{RESULTS AND DISCUSSION}

The computed $I_{d s}\left(V_{d s}, V_{g s}\right)$ characteristics are presented in figure 3 for both GaN and SiC MESFET. The obtained characteristics are almost identical in shape. For both structures, one observes that the output conductance is quite large and that the device is not completely pinched-off even at large negative gate bias. These two effects are mainly due to short channel effects and strong electron injection in the buffer layer. A part of the drain current flows through the buffer and increases with increasing drain voltage. This effect is more important at high $V_{g s}$, e.g. at $V_{g s}=-13 \mathrm{~V}$, the whole drain current flows entirely through the buffer. This is due to the low conduction band discontinuity at the interface between $\mathrm{GaN} / \mathrm{SiC}$ and their buffer layers. Nevertheless, for both structures, high drain current densities are obtained, confirming that GaN or SiC MESFETs are good candidates for high power applications. Moreover, due to the better electronic transport properties in $\mathrm{GaN}$, the GaN based MESFET exhibits a 70\% higher drain current density as compared with the SiC MESFET.

The effect of device length on output drain current have also been studied in figure 3. It is apparent from this figure, that higher drain current are reached as the device length is reduced as a result of the increase in longitudinal electric field and velocity overshoot effects. It follows that the electron transit time

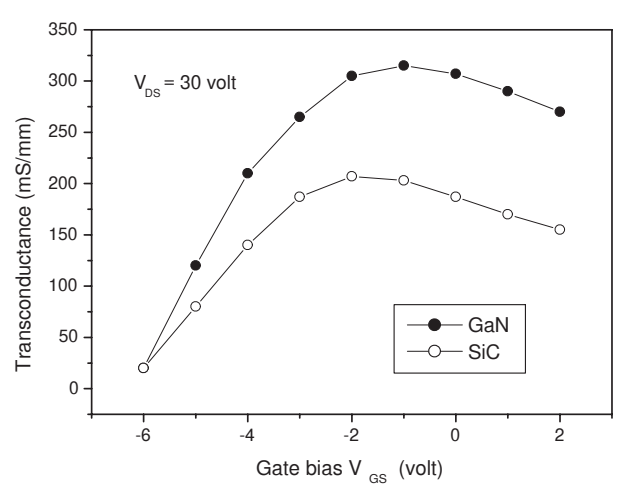

FIG. 4: Transconductance as a function of gate bias. Comparison of $\mathrm{GaN}$ and SiC MESFET. 


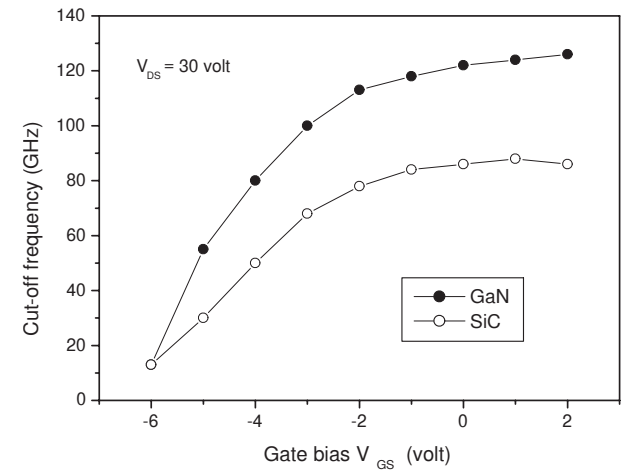

FIG. 5: Current gain cut-off frequency as a function of gate bias. Comparison of GaN and SiC MESFET.

under the gate is reduced in two ways; there is a reduction in the transit length and also the electron velocity is larger. The high value of the field at the source-end of the gate is responsible for the almost ballistic acceleration of the electrons as soon as they enter the channel region under the gate. As a result, shorter devices lead to higher cutoff frequencies but they are also expected to lead to smaller breakdown voltages.

Figures 5 and 6 present the evolution of transconductance $g_{m}$ (Fig. 5) and current gain cut-off frequency $f_{c}$ (Fig. 6) versus gate voltage $V_{g s}$, at $V_{d s}=30 \mathrm{~V}$, for both structures. Due to the better electronic properties and higher drain current den- sities, GaN MESFET exhibits $70 \%$ higher transconductance and cut-off frequency. For example, at $V_{g s}=0.2 \mathrm{~V}$, we obtained transconductance of $300 \mathrm{mS} \mathrm{mm} \mathrm{mm}^{-1}$ and cutoff frequency of $120 \mathrm{GHz}$ for the GaN MESFET, against $160 \mathrm{mS} \mathrm{mm}^{-1}$ and 85 $\mathrm{GHz}$ for the $\mathrm{SiC}$. These results show that GaN MESFETs may perform quite well for high frequency operations as well as for high power applications.

\section{CONCLUSIONS}

The Monte Carlo technique has been used to compare the potentialities of $\mathrm{GaN}$ and SiC MESFETs. We find that both materials are good candidates for high frequency and high power operations. At low operationg frequencies $\mathrm{GaN}$ drain $I V$ characteristics are superior to those of $\mathrm{SiC}$, possessing greater output resistances and higher gains. However, the $\mathrm{SiC}$ devices displayed substantially greater stability with respect to changes of voltage because of the ability of SiC to maintain a positive differential resistance at the drain contact due to ballistic effects under the gate.

\section{Acknowledgements}

The author wish to thank M. G. Paeezi for helpful comments and for critical reading of the manuscript.
[1] R. Mickevicius and J. H. Zhao, J. Appl. Phys. 83, 3161 (1998)

[2] R. J. Trew and M. W. Shin, Third Int. Conf. on Integrated Nonlinear Microwave and Millimeterwave Circuits Dig., 109 (1994)

[3] K. Dohnke, R. Rupp, D. Peters, J. Volkl and D. Stephani, Institute of Physics Conf. Series, IOP Publishing, Bristol, UK, 625 (1994)

[4] A. K. Agarwal, The Second Int. Electric Electronic Combat Vehicle Conf. AECV-II, Dearborn, MI, (1997)

[5] R. P. Joshi, J. Appl. Phys. 78, 5518 (1995)

[6] H. E. Nilsson, U. Sannemo and C. S. Petersson, J. Appl. Phys. 80, 3365 (1996)

[7] W. Fawcett, A. D. Boardman and S. J. Swain, J. Phys. Chem. Solids, 31, (1963)

[8] C. Jacoboni and P. Lugli, The Monte Carlo Method for semiconductor and Device Simulation, 1989, Springer-Verlag
[9] C. Moglestue, Monte Carlo Simulation of Semiconductor Devices, 1993, Chapman and Hall

[10] H. Arabshahi, Modern Physics Letters B. 20787 (2006)

[11] H. Arabshahi and M. H. Ghasemian, Modern Physics Letters B. 221397 (2006)

[12] H. Arabshahi, M. R. Benam and B. Salahi Modern Physics Letters B. 21, 1715 (2007)

[13] H. Arabshahi, Modern Physics Letters B. 21, 199 (2007)

[14] M. Walmsley and R. A. Abram, Comput. Math. Electr. Electron. Eng. 15, 31 (1996)

[15] J. B. Roldan, F. Gamiz, J. A. Lopez-Villanueva, and P. Cartujo, J. Electron Matter 26, 203 (1997)

[16] Y. C. Yeo, T. C. Chong and M. F. Li, J. Appl. Physics. 83, 1429 (1998) 